\title{
The Impact of Regular Cannabis and Methamphetamine Use on Psychological Distress and Self-Reported Mental Health Among Regular Ecstasy Users
}

\author{
L. Scott ${ }^{*}$ A. Roxburgh and L. Burns
}

National Drug and Alcohol Research Centre, University of New South Wales, Australia

Keywords: Ecstasy, cannabis, methamphetamine, mental health, depression, anxiety, drugs, K10, Kessler psychological distress scale, EDRS.

\section{INTRODUCTION}

Recent research has shown that large proportions of regular ecstasy users (REU) experience psychological distress, apparently independent of their ecstasy use [1]. It has been suggested that specific drugs, as well as polydrug use alone, may impact on psychological wellbeing among users [2]. There is some evidence that cannabis use is associated with psychological distress among $\operatorname{REU}[1,3]$. Methamphetamines, commonly used by REU [4], have also been associated with poor mental health outcomes [5]. This study aimed to examine the contribution of regular cannabis or methamphetamine use to levels of psychological distress and self-reported mental health problems among REU. were classified according to whether they regularly used cannabis or methamphetamines or had no additional regular illicit drug use. All eligible participants $(\mathrm{N}=1322)$ used drugs other than ecstasy. In addition to regular ecstasy use, 76 reported regular methamphetamine use, 464 reported regular cannabis use, and 135 reported both regular methamphetamine and cannabis use. The survey included: demographics, drug and alcohol use, self report measures of mental health and the Kessler Psychological Distress Scale (K10) (Fig. 1). Groups were compared using the Kruskal Wallis test with post-hoc analyses completed using Dunn's procedure and evaluated against corrected p-values.

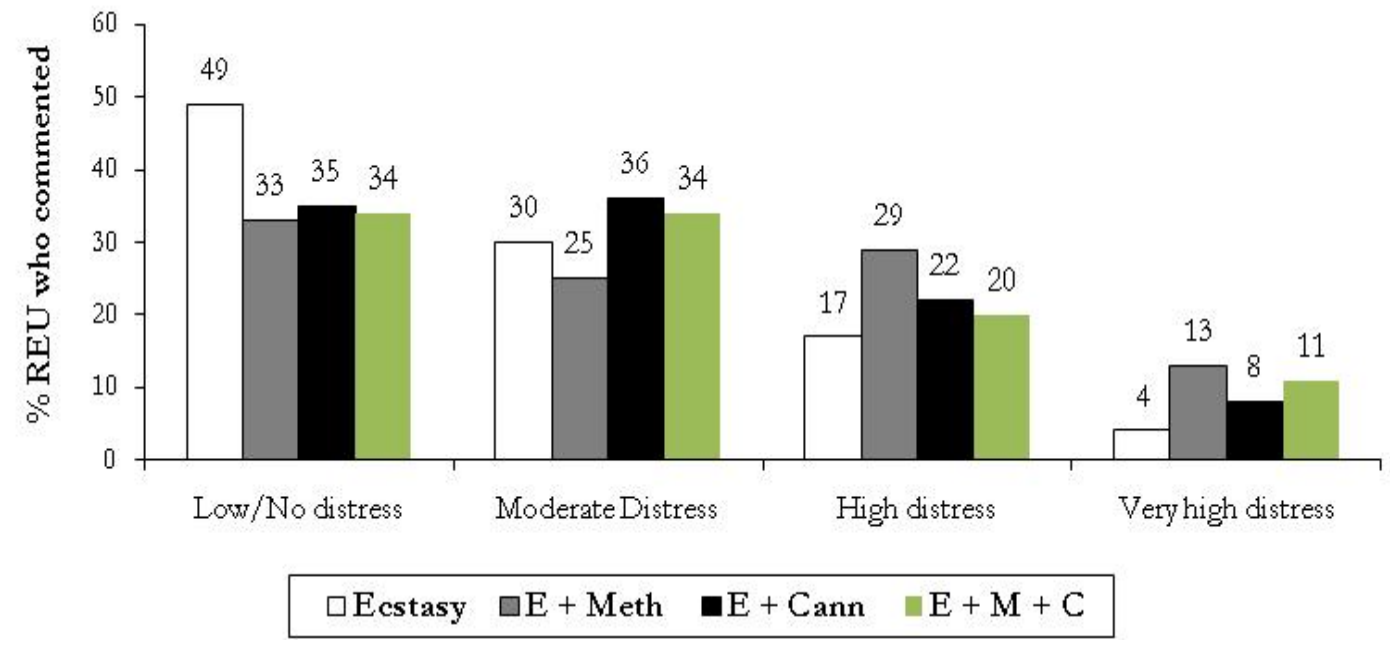

Fig. (1). Kessler Psychological Distress Scales (10 item) Scores for REU within each group.

\section{METHODS}

Data from the 2008 and 2009 Ecstasy and Related Drugs Reporting System (EDRS) were analysed. The EDRS is an annual, national, drug monitoring project that includes a cross-sectional survey of sentinel samples of REU. REU

*Address correspondence to this author at the National Drug and Alcohol Research Centre, University of New South Wales, Australia; Tel: 029385 0407; Fax: 029385 0222; E-mail: 1.scott@unsw.edu.au

\section{RESULTS}

REU who regularly used cannabis were significantly younger than their counterparts, had first used ecstasy at younger age and first reported regular ecstasy use at a younger age.

Regular methamphetamine use was associated with a higher number of days of use of ecstasy over the preceding six months. Levels of psychological distress were significantly higher among cannabis and methamphetamine 
using groups $\left(\chi^{2}=41.6, \mathrm{p}<0.001\right)$ than among REU who did not regularly use these drugs (Fig. 1). However, post-hoc analyses did not reveal any differences between these groups. Additionally, over one-quarter of the sample overall reported having experienced a mental health problem in the preceding six months. Self reported mental health problems were higher among REU who regularly used methamphetamine and/or cannabis, with depression most commonly reported, followed by anxiety.

\section{DISCUSSION}

These results support existing findings that cannabis use is associated with increased risk of psychological distress among REU. They also suggest that regular methamphetamine use may contribute to elevated psychological distress for this group. These initial investigations indicate that there may be no difference to the risk of elevated psychological distress associated with either cannabis or methamphetamine use or indeed regular use of both drugs among REU. However, differences in the frequency of ecstasy use and in various demographic variables were noted between the groups. Further analyses, will attempt to examine the relative contributions of cannabis and/or methamphetamine use while also controlling for these demographic variables and for differences in the frequency of ecstasy use.

\section{CONCLUSIONS}

These data indicate regular methamphetamine and/or cannabis use may confer additional risk for mental health problems among REU. Development of effective treatment strategies are clearly warranted and research which attempts to identify the additional risks associated with comorbid regular drug use may be useful in doing so.

\section{REFERENCES}

[1] George J, Kinner SA, Bruno R, Degenhardt L, Dunn $M$. Contextualising psychological distress among regular ecstasy users: the importance of sociodeographic factors and patterns of drug use. Drug Alcohol Rev 2010; 29(3): 243-9.

[2] Booth BM, Curran G, Han X, et al. Longitudinal relationship between psychological distress and multiple substance use: results from a three-year multisite natural-history study of rural stimulant users. J Stud Alcohol Drugs 2010; 71(2): 258-67.

[3] Daumann J, Hensen G, Thimm B, Rezk M, Till B, GouzoulizMayfrank E. Self-reported psychopathological symptoms in recreational ecstasy (MDMA) users are mainly associated with regular cannabis use: further evidence from a combined crosssectional/longitudinal investigation. Psychopharmacology 2004; 173: $398-404$.

[4] Sindicich N, Burns L. Australian trends in Ecstasy and related drug markets 2010. Findings from the Ecstasy and Related Drugs Reporting System (EDRS). Aust Drug Trends Series; No.46. NDARC: University of New South Wales, 2010.

[5] Darke S, Kaye S, McKetin R, Duflou J. Major physical and psychological harms of methamphetamine use. Drug Alcohol Rev 2008; 27(3): 253-62. 